\title{
Blocking SphK1/S1P/S1PR1 Signaling Pathway Alleviates Lung Injury Caused by Sepsis in Acute Ethanol Intoxication Mice
}

\author{
Liang Chen, ${ }^{1}$ Lingling $\mathrm{Li}^{1}{ }^{1}$ Yong Song $\oplus^{2,3}$ and Tangfeng $\mathrm{Lv}^{2,3}$
}

Received February 1 2021; accepted May 252021

\begin{abstract}
Acute ethanol intoxication increases the risk of sepsis and aggravates the symptoms of sepsis and lung injury. Therefore, this study aimed to explore whether sphingosine kinase 1 (SphK1)/sphingosine-1-phosphate (S1P)/S1P receptor 1 (S1PR1) signaling pathway functions in lung injury caused by acute ethanol intoxication-enhanced sepsis, as well as its underlying mechanism. The acute ethanol intoxication model was simulated by intraperitoneally administering mice with $32 \%$ ethanol solution, and cecal ligation and puncture (CLP) was used to construct the sepsis model. The lung tissue damage was observed by hematoxylin-eosin (H\&E) staining, and the wet-to-dry (W/D) ratio was used to evaluate the degree of pulmonary edema. Inflammatory cell counting and protein concentration in bronchoalveolar lavage fluid (BALF) were, respectively, detected by hemocytometer and bicinchoninic acid (BCA) method. The levels of tumor necrosis factor (TNF)- $\alpha$, interleukin (IL)-6, IL-1 $\beta$, and IL-18 in BALF were detected by their commercial enzyme-linked immunosorbent assay (ELISA) kits. The myeloperoxidase (MPO) activity and expression of apoptosis-related proteins and SphK1/S1P/S1PR1 pathway-related proteins were, respectively, analyzed by MPO ELISA kit and Western blot analysis. The cell apoptosis in lung tissues was observed by TUNEL assay. Acute ethanol intoxication (EtOH) decreased the survival rate of mice and exacerbated the lung injury caused by sepsis through increasing pulmonary vascular permeability, neutrophil infiltration, release of inflammatory factors, and cell apoptosis. In addition, EtOH could activate the SphK1/S1P/S1PR1 pathway in CLP mice. However, PF-543, as a specific inhibitor of SphK1, could partially reverse the deleterious effects on lung injury of CLP mice. PF-543 alleviated lung injury caused by sepsis in acute ethanol intoxication rats by suppressing the SphK1/S1P/S1PR1 signaling pathway.
\end{abstract}

KEY WORDS: SphK1/S1P/S1PR1 signaling pathway; lung injury; sepsis; acute ethanol intoxication.

\footnotetext{
${ }^{1}$ Department of Respiratory and Critical Care Medicine, The Affiliated No.1 People's Hospital of Nanjing Medical University, Huaian, 223300, Jiangsu, China

${ }^{2}$ Jinling Clinical Medical College, Nanjing Medical University, 305 Zhongshan East Road, Xuanwu District, Nanjing City, 210002, Jiangsu Province, China
}

\footnotetext{
${ }^{3}$ To whom correspondence should be addressed at Jinling Clinical Medical College, Nanjing Medical University, 305 Zhongshan East Road, Xuanwu District, Nanjing City, 210002, Jiangsu Province, China. E-mails: songyuanssyy@163.com; bairoushui@163.com
} 


\section{INTRODUCTION}

Sepsis is one of the main causes of death in intensive care unit (ICU) patients. Over $50 \%$ of patients with sepsis in ICU will suffer from acute lung injury (ALI) with a vastly high incidence and mortality rate [1]. Acute ethanol intoxication has been shown to have deleterious effects on outcomes in sepsis [2,3] and lung injury [4,5].

Sphingosine kinase-1 (SphK1) is an intracellular lipid enzyme that regulates cellular lipid metabolism and catalyzes the production of sphingosine 1-phosphate (S1P), which is directly involved in cellular signal transduction [6]. Intracellular S1P can also be transported to the extracellular domain by transporters on the cell membrane, and its binding to its own receptor indirectly activates intracellular cell transduction. The SPHK1-S1P-phosphate sphingosinol receptor (S1PR) signaling pathway is involved in the regulation of biological life activities such as cell proliferation, death, migration, and inflammation [7]. Existing evidence has emerged to suggest the essential roles of SphK1 and S1PR1 in inflammatory response [8]. $\mathrm{SphKl}$ was found to be involved in inflammatory responses in elderly rats [9]. The expression of S1PR1 in human gingival epithelial cells was upregulated after endotoxin stimulation, along with augmented contents of inflammatory factors [10]. Collectively, SphK1/S1P/S1PR1 pathway can be involved in inflammatory response, but its mechanism in lung injury caused by acute ethanol intoxication-enhanced sepsis is unknown.

The expression level of SphK1 mRNA in peripheral blood monocytes of patients with sepsis was higher, and inhibition of SphK1 could stabilize vascular endothelial cadherin and reduce microvascular leakage caused by sepsis [11]. The expression of SphK1 in the ileum of sepsis mice was increased by 6 times. SphK1 inhibitors reduced the production of pro-inflammatory cytokines and alleviated intestinal epithelial injury during sepsis [12]. N-3 polyunsaturated fatty acids inhibited SphK1 to improve parenteral nutrition and cecal ligation and puncture (CLP)-induced inflammation in sepsis rats [13]. Apigenin inhibited SphK1/S1P signaling pathway to alleviate heart injury in endotoxemic rats [14]. The upregulation of SphK1 was deemed as a key factor for the progression of ventilator-associated lung injury [15]. SphK1/S1P inhibitor combined with targeted drug therapy improved lung function in mice after cardiac death [16]. Alcohol intake caused pro-inflammatory changes in the intestinal immune cells and an increase in Th17 cells. S1P/S1PR1 signaling pathway was necessary in Th17 cell-mediated alcoholic liver disease formation, and in vivo intervention presented previously, i.e., blocking S1P/S1PR1 signaling pathway, could significantly reduce alcohol-induced inflammatory steatosis and liver injury [17].

Therefore, the present study was designed to investigate whether SphK1/S1P/S1PR1 signaling pathway can also function in lung injury caused by acute ethanol intoxication-enhanced sepsis, as well as its underlying mechanism.

\section{MATERIALS AND METHODS}

\section{Mouse Model of Acute Ethanol Intoxication and Sepsis}

Animal experiments were approved by the Animal Ethics Committee of Jinling Clinical Medical College of Nanjing Medical University. After a week of adjustable feeding, twenty male C57/BL6 mice (10-12 weeks) were administered with saline by gavage for $48 \mathrm{~h}$, and thirty male C57/BL6 mice (10-12 weeks) were administered with $32 \%$ ethanol solution $(6 \mathrm{~g} / \mathrm{kg})$ by gavage for $48 \mathrm{~h}$ [3]. In a preliminary experiment, we confirmed that mice did not die from binge ethanol administration over a 48 -h observation period. Twenty mice treated with saline were divided into two groups, including the sham group (performed sham-operation) and CLP group (performed cecal ligation and puncture) $(\mathrm{n}=10)$. Mice were subjected to CLP as described [18]. Thirty mice treated with $32 \%$ ethanol solution were divided into three groups, including EtOH group (performed sham operation), EtOH+CLP group (performed cecal ligation and puncture), and $\mathrm{EtOH}+\mathrm{CLP}+\mathrm{PF}-543$ group (performed cecal ligation and puncture and intraperitoneally injected $10 \mathrm{mg} / \mathrm{kg}$ PF-543 for 5 days) [11] $(\mathrm{n}=10)$. Immediately after anesthesia and exsanguination, lungs were collected and processed accordingly. PF-543, which is brought from MedChemExpress (Monmouth Junction, NJ), is a specific inhibitor of SphK1. This study was performed according to the National Institutes of Health Guide for Care and Use of Laboratory Animals.

\section{Hematoxylin-eosin (H\&E) Staining}

The lung tissue was fixed with $4 \%$ paraformaldehyde and then embedded with conventional dehydrated paraffin. Tissue sections that are $5 \mu \mathrm{m}$ were prepared, stained with hematoxylin and eosin, and observed by a light microscope. The H\&E procedure was performed as previously described [19]. 


\section{Detection of Wet-to-Dry (W/D) Ratio of Lung Tissue}

Determining the W/D ratio is the main indicator for assessing the extent of pulmonary edema. At the end of the experiment, the right lung was immediately removed and weighed to obtain the wet weight. Then, the lung was placed in an oven at $70^{\circ} \mathrm{C}$ and weighed approximately $24 \mathrm{~h}$ later. The dry weight was recorded when the measured value was constant.

\section{Inflammatory Cell Counting and Protein Concentration in Bronchoalveolar Lavage Fluid (BALF)}

The trachea was exposed, and the left lung was lavaged with sterile PBS for 3 times with $0.5-\mathrm{mL}$ PBS each time. The average liquid recovery after lavage was $>$ $70 \%$. The BALF was centrifuged at $2000 \mathrm{rmp}$ for $10 \mathrm{~min}$ at $4^{\circ} \mathrm{C}$, and the supernatant was stored at $-80^{\circ} \mathrm{C}$ for cytokine and protein analysis, and the cell mass was resuspended in PBS for cell counting. The total inflammatory cells in BALF were counted under a hemocytometer. A $100-\mu \mathrm{L}$ BALF was centrifuged onto slides by a Cytospin, which was dried, fixed, and stained with Wright stain solution in accordance with the instructions. The polymorphonuclear neutrophils (PMNs) were counted, and the percentage of neutrophils was calculated. The BALF supernatant was sufficiently mixed, and total protein concentration was determined by the bicinchoninic acid (BCA) method.

\section{Myeloperoxidase (MPO) Activity}

Lung tissue was homogenized, and the MPOcontaining supernatant was incubated for $30 \mathrm{~min}$ with $50 \mathrm{mmol} \mathrm{KPO}_{4}$ buffer containing $\mathrm{H}_{2} \mathrm{O}_{2}(1.5 \mathrm{mmol})$ and o-anisidine dihydrochloride (167 mg/mL, Sigma Aldrich). Finally, the enzyme activity was measured by a microplate reader in absorbance at $460 \mathrm{~nm}$.

\section{Detection of Inflammatory Cytokines}

The levels of tumor necrosis factor (TNF)- $\alpha$, interleukin (IL)-6, IL-1 $\beta$, and IL-18 in BALF supernatant were in turn detected by Mouse TNF- $\alpha$ ELISA Kit (cat. no. PT512; Beyotime), Mouse IL-6 ELISA Kit (cat. no. PI326; Beyotime), Mouse IL-1 $\beta$ ELISA Kit (cat. no. PI301; Beyotime), and Mouse IL-18 ELISA Kit (cat. no. PI553; Beyotime) according to the instructions.

\section{TUNEL Assay}

The lung tissue sections were incubated with protein kinase $\mathrm{K}$ for $30 \mathrm{~min}$. After rinsed with $\mathrm{PBS}$, terminal deoxyribonucleotide transferase (TdT) and deoxyuridine triphosphate (dUTP) labeled with luciferase were added to the sections. After reaction at $37^{\circ} \mathrm{C}$ for $1 \mathrm{~h}$, specific antibodies labeled with horseradish peroxidase were added to the sections, which were incubated at $37^{\circ} \mathrm{C}$ in the incubator for another $1 \mathrm{~h}$. Then, sections were treated with diaminobenzidine (DAB) at room temperature for 10 min, stained with 4',6-diamidino-2-phenylindole (DAPI), photographed, and quantified under a fluorescence microscope.

\section{Western Blot Analysis}

The lung tissue homogenate of mice in each group was lysed by RIPA lysate at $4^{\circ} \mathrm{C}$ for $30 \mathrm{~min}$ and centrifuged at $12000 \mathrm{rpm}$ for $20 \mathrm{~min}$ at $4^{\circ} \mathrm{C}$, and the total protein solution was obtained by collecting supernatant. The protein concentration of the samples was determined by the BCA method, and the $20-\mu \mathrm{g}$ protein samples were separated by SDS-PAGE electrophoresis and transferred to the PVDF membranes. They were then incubated with primary antibodies including Bcl-2 (ab182858; dilution, 1:2000; Abcam), Bax (ab182733; dilution, 1:2000; Abcam), ccaspase 3 (\#9661; dilution, 1:1000; Cell Signaling Technology), c-PARP (ab32064; dilution, 1:1000; Abcam), caspase 3 (\#9662; dilution, 1:1000; Cell Signaling Technology), PARP (ab191217; dilution, 1:1000; Abcam), SphK1 (cat. no. PA5-14068; dilution, 1:2000; Thermo Fisher Scientific), S1PR1 (cat. no. A70547-050; dilution, 1:1000; EpiGentek), and GAPDH (ab9485; dilution, 1:2500; Abcam) and incubated with a secondary antibody conjugated with horseradish peroxidase. Electrochemiluminescence (ECL) was used to present the bands of the targeted proteins in lung tissue.

\section{Reverse Transcription-Quantitative Polymerase Chain Reaction (RT-qPCR) Assay}

Total RNA of lung tissue homogenate of mice in each group was extracted using TRIzol reagent according to the manufacturer's protocol. The RNA extracts were reverse transcribed into cDNA and then quantified by SYBR Green assay (PowerTrack SYBR Green Master Mix) in the Agilent Mx3000P qPCR system. GAPDH was regarded as an internal reference. All primer sequences are presented as follows: Sphk1 forward 5'- 
GGAGGAGGCAGAGATAAC-3' and reverse 5'TTAGCCCATTCACCACTTCA-3'; S1PR1 forward 5'CCTCGGTGGTGTTCATTC-3' and reverse 5'GCAGGTTAGCTGTGTAGG-3'; GAPDH forward 5'GGTCTCCTCTGACTTCAACA-3' and reverse 5'AGCCAAATTCGTTGTCATAC-3'. The data were analyzed by $2^{-\Delta \Delta \mathrm{Cq}}$ method [20].

\section{Detection of SphK1 and S1P}

After pretreatment of removing the blood from the lung tissue, tissue was homogenized and centrifuged at $12000 \mathrm{rpm}$ to obtain the SphK1 and S1P-containing supernatant. According to the instructions of the SPHK1 Activity Assay kit (cat. no. KA0906; Abnova) and S1P ELISA kit (cat. no. K-1900; Echelon Biosciences), the substrate required for enzyme reaction was added to the supernatant, which was incubated at $30^{\circ} \mathrm{C}$ for $3 \mathrm{~min}$, and the levels of $\mathrm{SphK1}$ and S1P were detected by a microplate reader.

\section{Statistical Analysis}

SPSS 20.0 software was used for data processing. Data were represented by mean \pm standard deviation. One-way analysis of variance and LSD multiple comparison were used to compare the differences between groups. $P<0.05$ was statistically significant.

\section{RESULTS}

\section{PF-543 Improves the Survival Rate of CLP Mice After} Acute Ethanol Intoxication and Lung Injury

The sham operation and acute ethanol intoxication did not virtually affect the rat survival rate. CLP decreased the survival rate of mice in the first 3 days, while combined treatment with CLP and acute ethanol intoxication decreased their survival rate more significantly until the fourth day and sustained at 30\%. PF-543 improved the survival rate of CLP mice after acute ethanol intoxication, which was decreased in the first 2 days and then sustained at $70 \%$ as the final outcome (Fig. 1A). The acute ethanol intoxication to a slighter extent damaged the lung tissues than mere CLP treatment. In the EtOH+CLP group, the alveolar wall was thickened, and a large number of inflammatory cells were infiltrated in the interstitial lung, which could be obviously alleviated by PF-543 (Fig. 1B).

\author{
PF-543 Decreases the Pulmonary Vascular \\ Permeability and Neutrophil Infiltration in the Lungs \\ of CLP Mice After Acute Ethanol Intoxication
}

CLP increased the W/D value than that in the EtOH group, which was further increased in the $\mathrm{EtOH}+\mathrm{CLP}$ group, and PF-543 decreased the W/D value in CLP mice after acute ethanol intoxication (Fig. 2A). The protein concentration and neutrophils in mice BALF of EtOH group were increased while not obviously different from that of the sham group. CLP increased the protein concentration and neutrophils in mice BALF, and the protein concentration and neutrophils in mice BALF were further increased in the EtOH+CLP group. PF-543 could decrease the protein concentration and neutrophils in BALF of CLP mice after acute ethanol intoxication (Fig. 2B and 2C). CLP increased the MPO activity than that in the EtOH group, and MPO activity was further increased in the EtOH+CLP group, which was decreased by PF-543 (Fig. 2D).

\section{PF-543 Decreases the Release of Inflammatory Factors in the Lungs of CLP Mice After Acute Ethanol Intoxication}

The expression levels of TNF- $\alpha$, IL-6, IL- $1 \beta$, and IL18 in mice BALF were increased after CLP operation than those in the EtOH group. The levels of inflammatory factors were higher in the EtOH+CLP group than that in the CLP group, which were partially reversed by PF-543 (Fig.3).

\section{PF-543 Decreases the Cell Apoptosis in the Lungs of CLP Mice After Acute Ethanol Intoxication}

Compared with the EtOH group, cell apoptosis in the lungs of CLP mice was increased, which was further increased in the lungs of CLP mice after acute ethanol intoxication. PF-543 significantly suppressed the cell apoptosis in the lungs of CLP mice after acute ethanol intoxication (Fig. 4A). The expression of Bax, cleaved-caspase $3 /$ caspase 3, and cleaved-PARP/PARP was gradually increased, and Bcl-2 expression was gradually decreased in lung tissues of mice in the EtOH group, CLP group, and EtOH+CLP group. The above protein expression in lung tissues of mice could be partially reversed by the treatment of PF-543 (Fig. 4B).

\section{PF-543 Blocks the SphK1/S1P/S1PR1 Signaling Pathway}

The SphK1 activity and S1P level were increased in CLP mice compared with the EtOH group. 

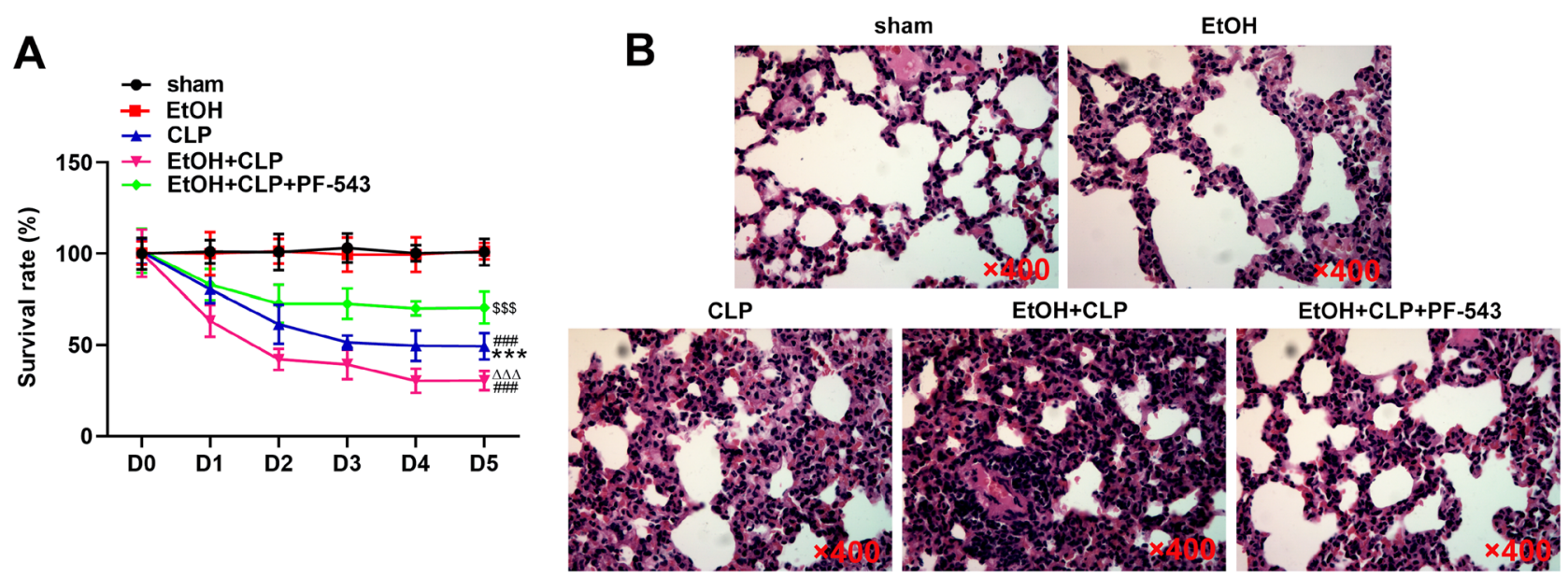

Fig 1. PF-543 improves the survival rate of CLP mice after acute ethanol intoxication and lung injury. A The survival rate of mice was recorded after CLP treatment. B Pulmonary pathology changes were observed by H\&E staining. ${ }^{*} * P<0.001$ vs. sham group. ${ }^{\# \#} P<0.001$ vs. EtOH group. ${ }^{\Delta \Lambda} P<0.001$ vs. CLP group. ${ }^{\$ \$} P<0.001$ vs. EtOH+CLP group.

The combined operation of acute ethanol intoxication and CLP could further increase the SphK1 activity and S1P level, which was partially inversed by PF543 (Fig. 5A and 5B). The mRNA and protein expression of SphK1 and S1PR1 in CLP mice was increased compared with that in the EtOH group, which was further increased after the additional operation of acute ethanol intoxication (Fig. $5 \mathrm{C}$ and Fig. 5D). PF-543 could not obviously decrease the mRNA and protein expression of SphK1 and S1PR1 in CLP mice with acute ethanol intoxication.

\section{DISCUSSION}

A clinical study showed that alcoholics were easier to get sepsis and thus have a higher mortality rate than that of nonalcoholics with sepsis [21]. Moreover, alcohol abuse was a leading risk factor for mortality, and about 100,000 people died from alcohol abuse every year [21, 22]. In addition, ethanol consumption could increase the incidence and severity of natural infections in humans and experimental animals $[22,23]$. Studies related to humans and animals unequivocally indicated that acute alcohol
A

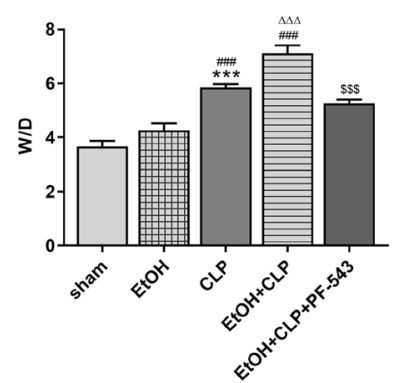

B

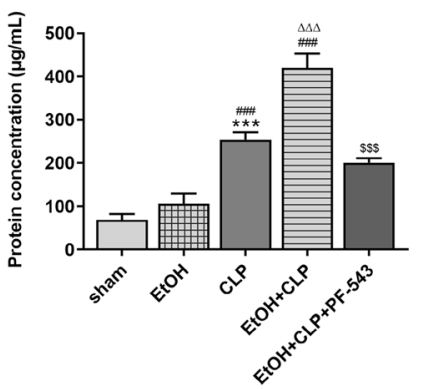

C

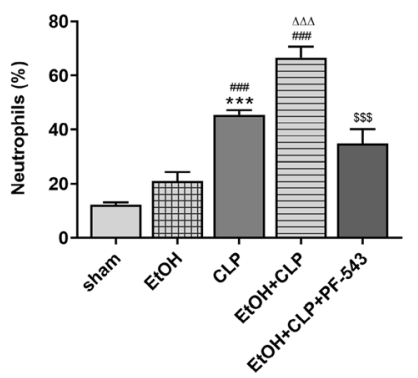

D

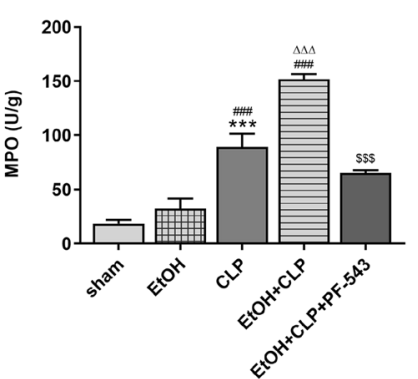

Fig. 2. PF-543 improves the pulmonary vascular permeability and neutrophil infiltration in the lungs of CLP mice after acute ethanol intoxication. A W/D values of lung tissues were recorded to evaluate the edema degree. B Protein concentration in BALF was detected by BCA assay. C The proportion of neutrophils in total inflammatory cells in BALF was calculated under a hemocytometer. D MPO activity in lung tissues was determined by its ELISA assay. ${ }^{* * *} P<0.001$ vs. sham group. ${ }^{\# \#} P<0.001$ vs. EtOH group. ${ }^{\Delta \Lambda} P<0.001$ vs. CLP group. ${ }^{\$ \$} P<0.001$ vs. EtOH+CLP group. 

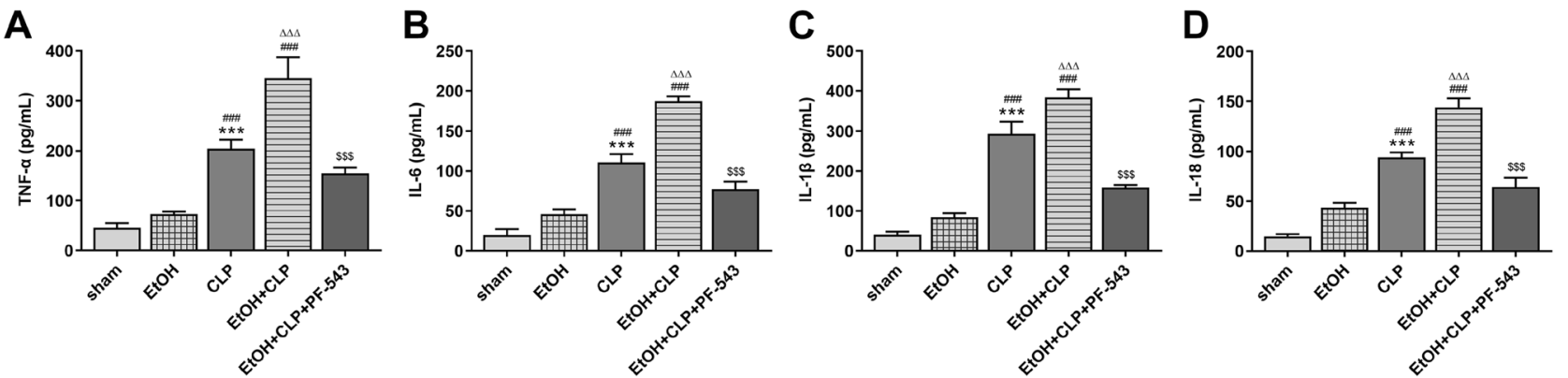

Fig. 3. PF- 543 decreases the release of inflammatory factors in the lungs of CLP mice after acute ethanol intoxication. The expression levels of TNF- $\alpha$ (A), IL-6 (B), IL-1 $\beta$ (C), and IL-18 (D) in lung tissues were determined by respective ELISA kit. ${ }^{* * *} P<0.001$ vs. sham group. ${ }^{\# \#} P<0.001$ vs. EtOH group. ${ }^{\Delta \Lambda \Lambda} P<0.001$ vs. CLP group. ${ }^{\$ \$} P<0.001$ vs. EtOH+CLP group.

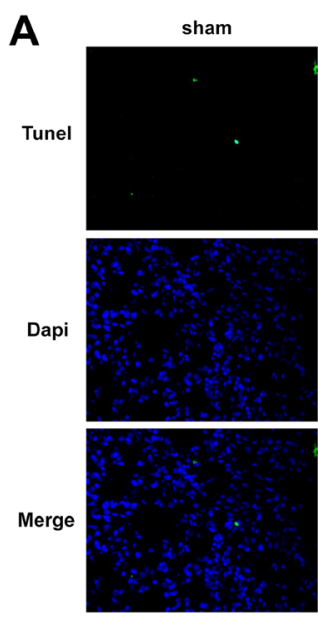

B

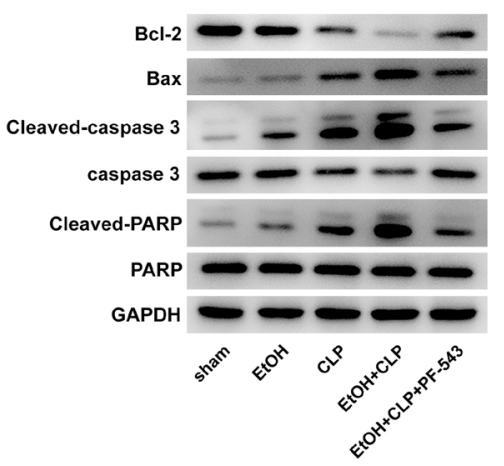

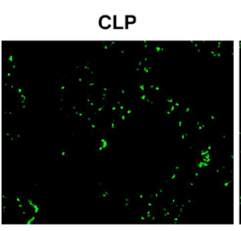
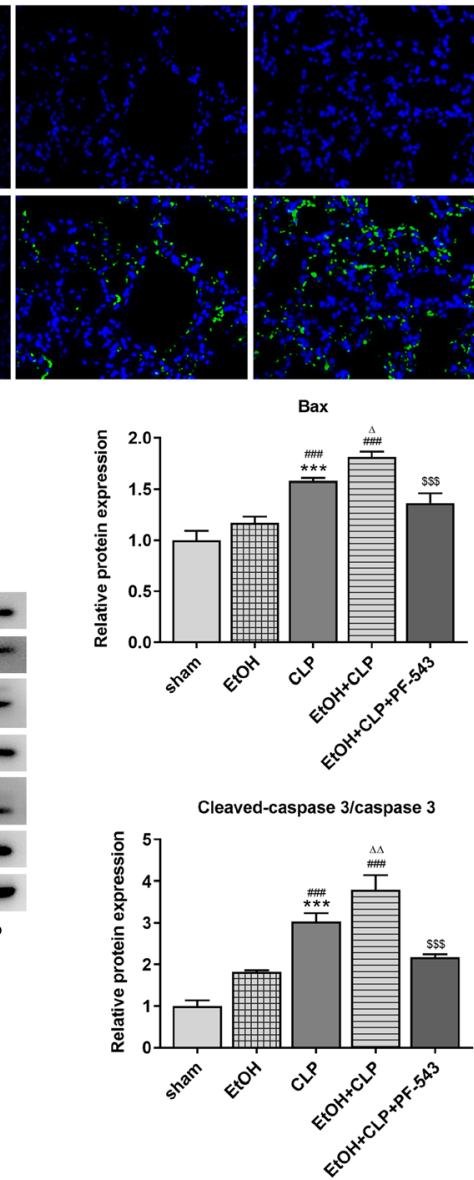

$\mathrm{EtOH}+\mathrm{CLP}+\mathrm{PF}-543$
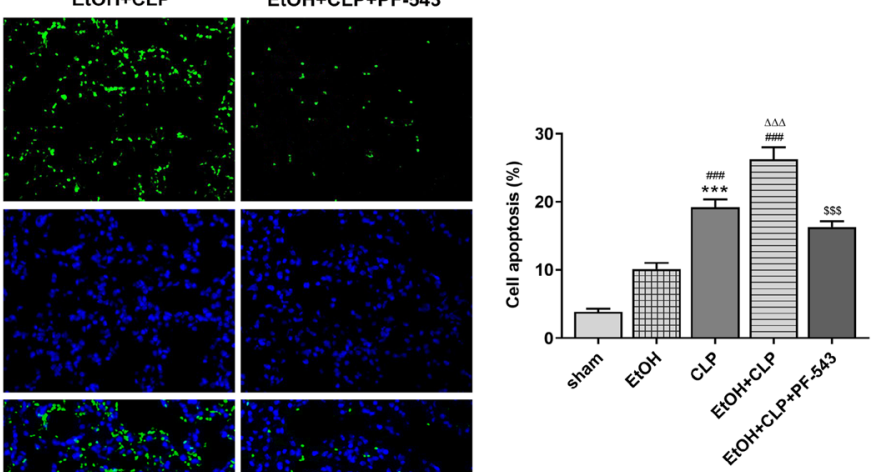

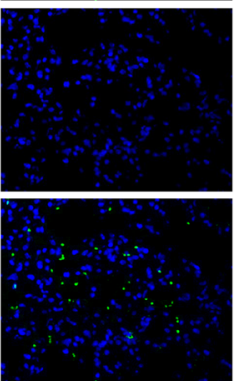

Bcl-2
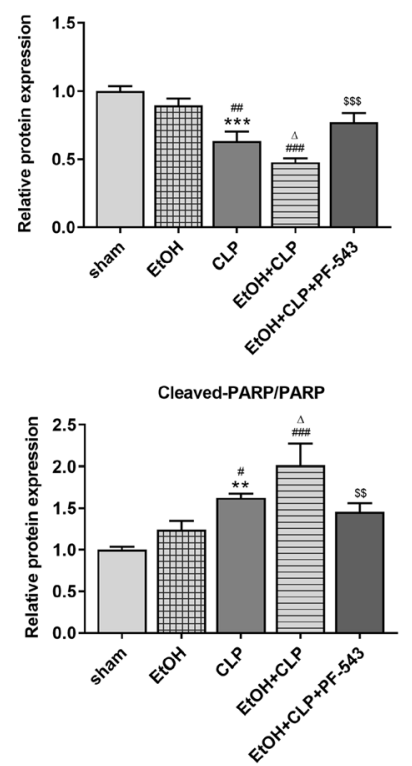

Fig. 4. PF-543 decreases the cell apoptosis in the lungs of CLP mice after acute ethanol intoxication. A The cell apoptosis in lung tissues was analyzed by TUNEL assay. B The apoptosis-related proteins expression was detected by Western blot. $* * P<0.01$ and $* * * P<0.001$ vs. sham group. ${ }^{\#} P<0.01$ and ${ }^{\# \# \#} P<0.001$ vs. EtOH group. ${ }^{\Delta} P<0.05$ and ${ }^{\Delta \Lambda} P<0.01$ vs. CLP group. ${ }^{\$ \$} P<0.01$ and ${ }^{\$ \$} P<0.001$ vs. EtOH+CLP group. 
A

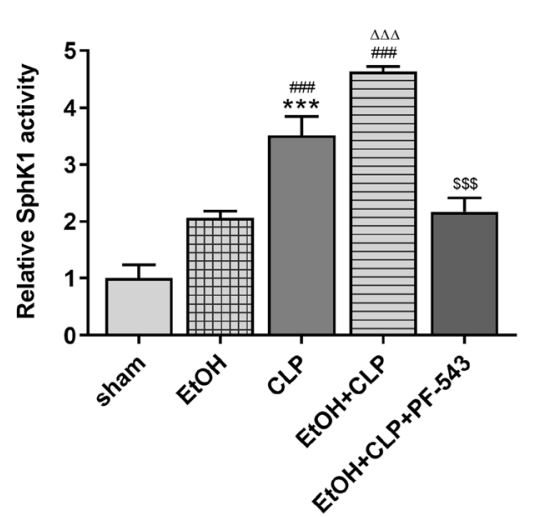

C

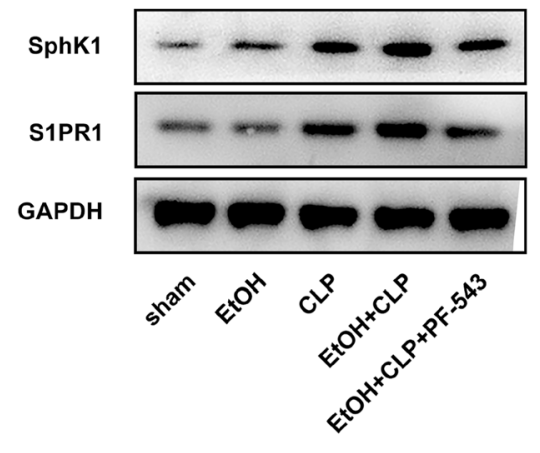

SphK1

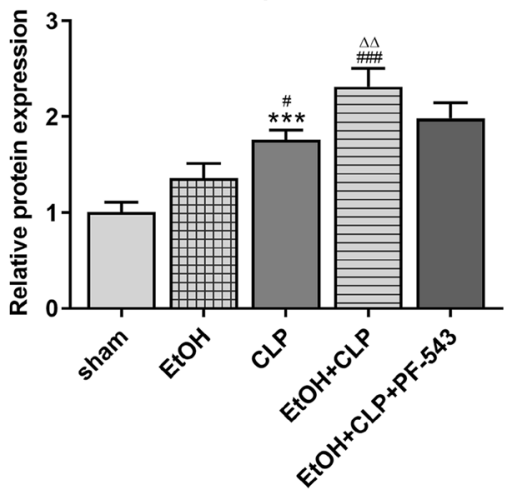

D

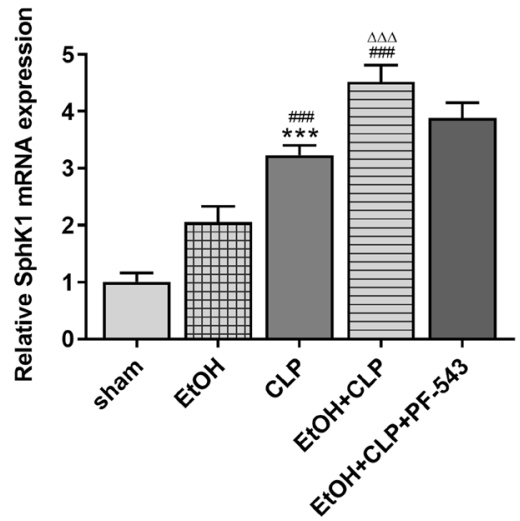

B

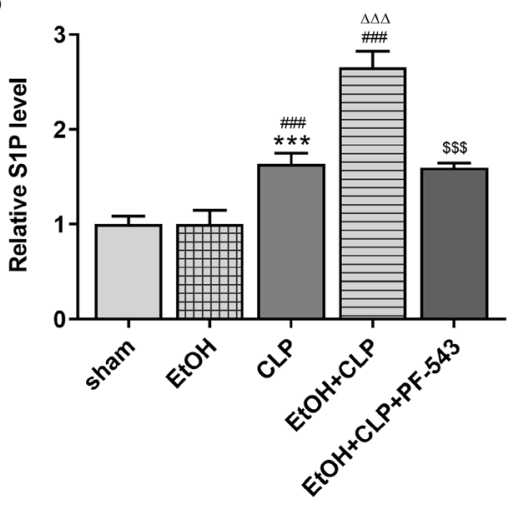

S1PR1
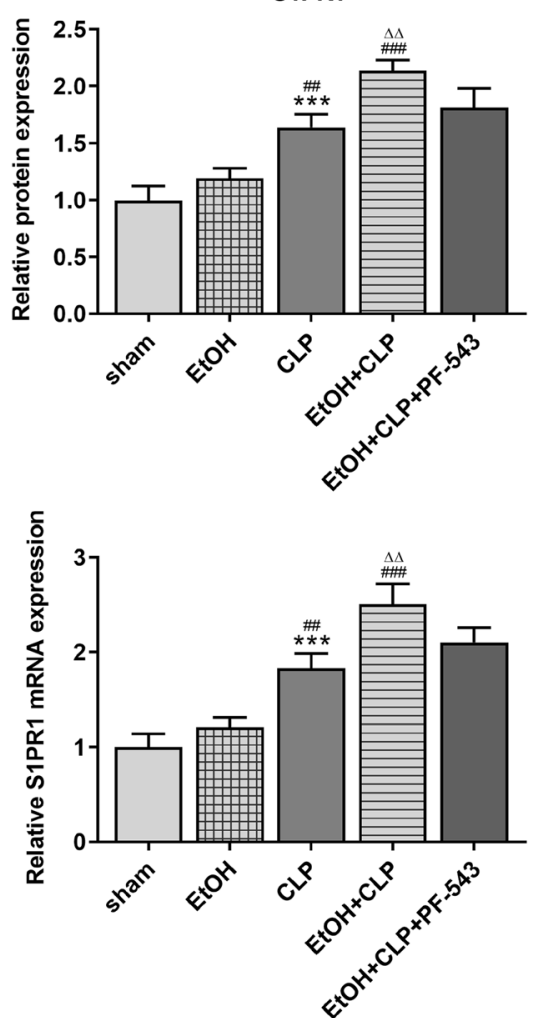

Fig. 5. PF-543 blocks the SphK1/S1P/S1PR1 signaling pathway. The SphK1 activity (A) and S1P level (B) in lung tissues were detected by respective kit. C The protein expression of SphK1 and S1PR1 in lung tissues was determined by Western blot. D The mRNA expression of SphK1 and S1PR1 in lung tissues

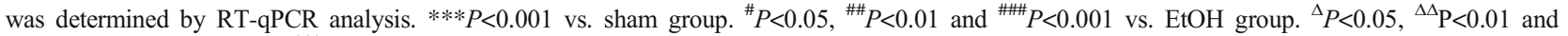
${ }^{\triangle \triangle \triangle} P<0.001$ vs. CLP group. ${ }^{\$ \$} P<0.001$ vs. EtOH+CLP group.

intoxication was a leading risk factor for mortality associated with sepsis [2, 24-26]. The present study indicated that CLP decreased the mouse survival rate and caused significant lung injury by pulmonary edema, inflammation, oxidative stress, and cell apoptosis of lung tissues in CLP mice. In addition, the lung injury caused by CLP was further aggravated when mice were in the state of acute ethanol intoxication at first.

S1P is a multipotent signaling lipid molecule widely present in the blood, lymph, and lymphatic tissue channels. It can be used as the second messenger to transmit signals in cells, and it can also interact with the corresponding $\mathrm{G}$ 
protein-coupled receptors on cell membranes as the first messenger to participate in biological effects including cell proliferation, migration, apoptosis, angiogenesis, and immune response [27-29]. S1P was the sphingolipid metabolite of mast cells, which induced the activation of mast cells. After activation, mast cells degranulated and released bioactive substances, leading to asthmatic airway inflammation [30]. Hyperglycemia, late glycosylation end products, hypoxia, and oxidative stress could activate SphK1, leading to increased production of S1P that could further promote cell proliferation and expression of the adhesion molecules and TGF- $\beta$ by binding to the receptor and induce inflammation in the kidney and nephropathy [3133]. Here, we revealed that CLP increased S1P level, which was further increased by additional operation of acute ethanol intoxication. The S1P level was positive with the levels of oxidative stress and inflammation in BALF and lung tissues. FTY 720, an S1P receptor agonist that can be combined with all S1PRs except S1PR2, could alleviate S1P-induced renal injury via decreasing inflammatory cytokines [34]. FTY 720 improved the inflammatory response of asthma and pulmonary histopathological changes by inhibiting the S1P1/WNT1/ RAC1/P38-MAPK pathway [35]. S1P promoted the inflammation induced by the activation of the $\beta 1$ adrenergic receptor in the cardiomyocytes, and FTY720 improved chronic cardiac inflammation and cardiac remodeling and dysfunction in vivo by suppressing the cardiac SphK1/S1P/S1PR1 signaling pathway [36]. By inhibiting pro-inflammatory cytokines (TNF- $\alpha$, IL-1 $\beta$, and IL-6), SphK inhibitors reduced polymorphic inflammatory cells in the placental vestibule, thereby preventing lipopolysaccharide-induced preterm birth [37]. SphK1-derived S1P promoted S1PR1 expression, forming a SphK1/S1P/S1PR1 amplification feedback loop. STAT3 is a transcription factor for the S1PR1 gene, and upregulated S1PR1 expression promoted the expression of STAT3 and IL-6 to accelerate the tumor growth and metastasis in a STAT3-dependent manner [38]. Liang et al. in Cancer Cell demonstrated that SphK1-mediated upregulation of S1P drove a persistent NFKB/IL-6/STAT3/S1PR1 amplification loop that was critical for the development of chronic colitis and colitis-associated cancer [39]. In this study, PF-543, as a specific inhibitor of SphK1, improved pulmonary vascular permeability, neutrophil infiltration, inflammation, oxidative stress, and cell apoptosis to alleviate lung injury by inhibiting the expression of S1P and S1PR1.
In conclusion, the present study demonstrated that acute ethanol intoxication further decreased the survival rate and increased the pulmonary edema, inflammation, oxidative stress, and cell apoptosis of lung tissues in CLP mice. PF-543 could significantly reverse the lung injury caused by CLP and acute ethanol intoxication. These findings reveal a novel mechanism underlying the acute ethanol intoxication-induced sepsis and a guiding significance for drug research and development.

\section{AUTHOR CONTRIBUTION}

Liang Chen: project development, data analysis and collection, manuscript writing

Lingling Li: project development, data analysis and collection, manuscript writing

Yong Song: project development, manuscript editing

Tangfeng Lv: project development, manuscript editing

\section{DATA AVAILABILITY}

The datasets used and/or analyzed during the current study are available from the corresponding author on reasonable request.DECLARATIONS

Ethics Approval and Consent to Participate. Animal experiments were approved by the Animal Ethics Committee of Jinling Clinical Medical College of Nanjing Medical University. The study was conducted in accordance with ARRIVE guidelines.

Consent for Publication. Not applicable.

Competing interests. The authors declare no competing interests.

\section{REFERENCES}

1. Jeganathan, N., S. Yau, N. Ahuja, D. Otu, B. Stein, L. Fogg, and R. Balk. 2017. The characteristics and impact of source of infection on sepsis-related ICU outcomes. Journal of Critical Care 41: 170-176.

2. Pruett, S.B., Q. Zheng, R. Fan, K. Matthews, and C. Schwab. 2004. Ethanol suppresses cytokine responses induced through toll-like receptors as well as innate resistance to escherichia coli in a mouse model for binge drinking. Alcohol (Fayetteville, NY) 33: 147-155.

3. Pruett, S.B., R. Fan, B. Cheng, M. Glover, W. Tan, and X. Deng. 2010. Innate immunity and inflammation in sepsis: mechanisms of suppressed host resistance in mice treated with ethanol in a binge- 
drinking model. Toxicological Sciences : An Official Journal of the Society of Toxicology 117: 314-324.

4. Yeligar, S.M., M.M. Chen, E.J. Kovacs, J.H. Sisson, E.L. Burnham, and L.A. Brown. 2016. Alcohol and lung injury and immunity. Alcohol (Favetteville, NY) 55: 51-59.

5. Mehta, A.J., S.M. Yeligar, L. Elon, L.A. Brown, and D.M. Guidot. 2013. Alcoholism causes alveolar macrophage zinc deficiency and immune dysfunction. American Journal of Respiratory and Critical Care Medicine 188: 716-723.

6. Spiegel, S., and S. Milstien. 2003. Sphingosine-1-phosphate: an enigmatic signalling lipid. Nature Reviews. Molecular Cell Biology 4: $397-407$

7. Proia, R.L., and T. Hla. 2015. Emerging biology of sphingosine-1phosphate: its role in pathogenesis and therapy. The Journal of Clinical Investigation 125: 1379-1387.

8. Rosen, H., R.C. Stevens, M. Hanson, E. Roberts, and M.B. Oldstone. 2013. Sphingosine-1-phosphate and its receptors: structure, signaling, and influence. Annual Review of Biochemistry 82: 637-662.

9. Lufrano, M., A. Jacob, M. Zhou, and P. Wang. 2013. Sphingosine kinase-1 mediates endotoxemia-induced hyperinflammation in aged animals. Molecular Medicine Reports 8: 645-649.

10. Eskan, M.A., B.G. Rose, M.R. Benakanakere, Q. Zeng, D. Fujioka, M.H. Martin, et al. 2008. TLR4 and S1P receptors cooperate to enhance inflammatory cytokine production in human gingival epithelial cells. European Journal of Immunology 38: 1138-1147.

11. Zhong, M., W. Wu, Y. Wang, H. Mao, J. Song, S. Chen, and D. Zhu. 2020. Inhibition of sphingosine kinase 1 attenuates sepsisinduced microvascular leakage via inhibiting macrophage NLRP3 inflammasome activation in mice. Anesthesiology 132: 1503-1515.

12. Ugwu, F.N., and J. Ho. 2019. Preclinical evidence of sphingosine kinase 1 inhibition in alleviation of intestinal epithelial injury in polymicrobial sepsis. Inflammation Research : Official Journal of the European Histamine Research Society [et al] 68: 723-726.

13. Tian, T., Y. Zhao, Q. Huang, and J. Li. 2016. n-3 polyunsaturated fatty acids improve inflammation via inhibiting sphingosine kinase 1 in a rat model of parenteral nutrition and CLP-induced sepsis. Lipids 51: 271 .

14. Zhang, T., T. Yan, J. Du, S. Wang, and H. Yang. 2015. Apigenin attenuates heart injury in lipopolysaccharide-induced endotoxemic model by suppressing sphingosine kinase 1 /sphingosine 1 phosphate signaling pathway. Chemico-Biological Interactions 233: 46-55.

15. Wang, Y., T.T. Gao, D.F. Xu, X.Y. Zhu, W.W. Dong, Z. Lv, Y.J. Liu, and L. Jiang. 2019. Upregulation of sphingosine kinase 1 contributes to ventilator-associated lung injury in a two-hit model. International Journal of Molecular Medicine 44: 2077-2090.

16. Mehaffey, J.H., E.J. Charles, A.K. Narahari, S. Schubert, V.E. Laubach, N.R. Teman, K.R. Lynch, I.L. Kron, and A.K. Sharma. 2018. Increasing circulating sphingosine-1-phosphate attenuates lung injury during ex vivo lung perfusion. The Journal of Thoracic and Cardiovascular Surgery 156: 910-917.

17. Chu S, Sun R, Gu X, Chen L, Liu M, Guo H, et al. 2021. Inhibition of sphingosine-1-phosphate-induced Th17 cells ameliorates alcoholic steatohepatitis in mice. Hepatology 73: 952-967.

18. Sharma, D., A. Malik, N. Packiriswamy, M.D. Steury, and N. Parameswaran. 2018. Poly(I:C) priming exacerbates cecal ligation and puncture-induced polymicrobial sepsis in mice. Inflammation 41: 328-336.

19. Wen, H., H. Zhang, W. Wang, and Y. Li. 2020. Tetrahydropalmatine protects against acute lung injury induced by limb ischemia/reperfusion through restoring PI3K/AKT/
mTOR-mediated autophagy in rats. Pulmonary Pharmacology \& Therapeutics 64: 101947.

20. Livak, K.J., and T.D. Schmittgen. 2001. Analysis of relative gene expression data using real-time quantitative PCR and the 2(-delta delta C(T)) method. Methods 25: 402-408.

21. Burnham, E.L., R. Halkar, M. Burks, and M. Moss. 2009. The effects of alcohol abuse on pulmonary alveolar-capillary barrier function in humans. Alcohol and Alcoholism 44: 8-12.

22. von Dossow, V., C. Schilling, S. Beller, O.V. Hein, C. von Heymann, W.J. Kox, et al. 2004. Altered immune parameters in chronic alcoholic patients at the onset of infection and of septic shock. Critical Care (London, England) 8: R312-R321.

23. Rittirsch, D., M.A. Flierl, and P.A. Ward. 2008. Harmful molecular mechanisms in sepsis. Nature Reviews. Immunology 8: 776-787.

24. Huttunen, R., J. Laine, J. Lumio, R. Vuento, and J. Syrjänen. 2007. Obesity and smoking are factors associated with poor prognosis in patients with bacteraemia. BMC Infectious Diseases 7: 13.

25. McGill, V., A. Kowal-Vern, S.G. Fisher, S. Kahn, and R.L. Gamelli. 1995. The impact of substance use on mortality and morbidity from thermal injury. The Journal of Trauma 38: 931-934.

26. Woodman, G.E., T.C. Fabian, J.D. Beard, and K.G. Proctor. 1996. Actions of acute ethanol intoxication on cardiopulmonary function after an endotoxin challenge. Surgery 120: 80-92.

27. Spiegel, S., and S. Milstien. 2000. Functions of a new family of sphingosine-1-phosphate receptors. Biochimica et Biophysica Acta 1484: 107-116.

28. Hla, T., M.J. Lee, N. Ancellin, J.H. Paik, and M.J. Kluk. 2001. Lysophospholipids-receptor revelations. Science 294: 1875-1878.

29. Pyne, S., and N. Pyne. 2000. Sphingosine 1-phosphate signalling via the endothelial differentiation gene family of G-protein-coupled receptors. Pharmacology \& Therapeutics 88: 115-131.

30. Galli, S.J. 2017. Mast cells and KIT as potential therapeutic targets in severe asthma. The New England Journal of Medicine 376: 19831984.

31. Wang, L., X.P. Xing, A. Holmes, C. Wadham, J.R. Gamble, M.A. Vadas, and P. Xia. 2005. Activation of the sphingosine kinasesignaling pathway by high glucose mediates the proinflammatory phenotype of endothelial cells. Circulation Research 97: 891-899.

32. You, B., A. Ren, G. Yan, and J. Sun. 2007. Activation of sphingosine kinase-1 mediates inhibition of vascular smooth muscle cell apoptosis by hyperglycemia. Diabetes 56: 1445-1453.

33. Xin, C., S. Ren, B. Kleuser, S. Shabahang, W. Eberhardt, H. Radeke, M. Schäfer-Korting, J. Pfeilschifter, and A. Huwiler. 2004. Sphingosine 1-phosphate cross-activates the Smad signaling cascade and mimics transforming growth factor-beta-induced cell responses. The Journal of Biological Chemistry 279: 35255-35262.

34. Su, K., P. Zeng, W. Liang, Z. Luo, Y. Wang, X. Lv, et al. 2017. FTY720 Attenuates angiotensin II-induced podocyte damage via inhibiting inflammatory cytokines. Mediators of Inflammation 2017: 3701385 .

35. Liu, H.Y., W.D. Liu, Z.A. Chen, G. Gao, and G.H. Yan. 2020. FTY-720 regulated airway inflammation in asthmatic mice via S1P1 receptor. Chinese Journal of Immunity 36: 1419-1421+1432.

36. Zhang, F., Y. Xia, W. Yan, H. Zhang, F. Zhou, S. Zhao, W. Wang, D. Zhu, C. Xin, Y. Lee, L. Zhang, Y. He, E. Gao, and L. Tao. 2016. Sphingosine 1-phosphate signaling contributes to cardiac inflammation, dysfunction, and remodeling following myocardial infarction. American Journal of Physiology. Heart and Circulatory Physiology 310: $\mathrm{H} 250-\mathrm{H} 261$.

37. Vyas, V., C.R. Ashby Jr., N.S. Olgun, S. Sundaram, O. Salami, S. Munnangi, R. Pekson, P. Mahajan, and S.E. Reznik. 2015. Inhibition of sphingosine kinase prevents lipopolysaccharide-induced 
preterm birth and suppresses proinflammatory responses in a murine model. The American Journal of Pathology 185: 862-869.

38. Lee, H., J. Deng, M. Kujawski, C. Yang, Y. Liu, A. Herrmann, M. Kortylewski, D. Horne, G. Somlo, S. Forman, R. Jove, and H. Yu. 2010. STAT3-induced S1PR1 expression is crucial for persistent STAT3 activation in tumors. Nature Medicine 16: 1421-1428.
39. Liang, J., M. Nagahashi, E.Y. Kim, K.B. Harikumar, A. Yamada, W.C. Huang, N.C. Hait, J.C. Allegood, M.M. Price, D. Avni, K. Takabe, T. Kordula, S. Milstien, and S. Spiegel. 2013. Sphingosine1-phosphate links persistent STAT3 activation, chronic intestinal inflammation, and development of colitis-associated cancer. Cancer Cell 23: 107-120. 